\title{
On New Approaches of Assessing Network Vulnerability: Hardness and Approximation
}

\author{
Thang N. Dinh, Ying Xuan, My T. Thai, Member, IEEE, Panos M. Pardalos, Member, IEEE, \\ Taieb Znati, Member, IEEE
}

\begin{abstract}
Society relies heavily on its networked physical infrastructure and information systems. Accurately assessing the vulnerability of these systems against disruptive events is vital for planning and risk management. Existing approaches to vulnerability assessments of large-scale systems mainly focus on investigating inhomogeneous properties of the underlying graph elements. These measures and the associated heuristic solutions are limited in evaluating the vulnerability of large-scale network topologies. Furthermore, these approaches often fail to provide performance guarantees of the proposed solutions. In this paper, we propose a vulnerability measure, pairwise connectivity, and use it to formulate network vulnerability assessment as a graph-theoretical optimization problem, referred to as $\beta$-disruptor. The objective is to identify the minimum set of critical network elements, namely nodes and edges, whose removal results in a specific degradation of the network global pairwise connectivity. We prove the NP-Completeness and inapproximability of this problem, and propose an $O(\log n \log \log n)$ pseudoapproximation algorithm to computing the set of critical nodes and an $O\left(\log ^{1.5} n\right)$ pseudo-approximation algorithm for computing the set of critical edges. The results of an extensive simulation-based experiment show the feasibility of our proposed vulnerability assessment framework and the efficiency of the proposed approximation algorithms in comparison with other approaches.
\end{abstract}

Index Terms-Network vulnerability, Pairwise connectivity, Hardness, Approximation algorithm.

\section{INTRODUCTION}

OnNectivity plays a vital role in network performance and is fundamental to vulnerability assessment. Disruptive events, ranging from natural disasters to malicious attacks, can drastically compromise the network's ability to meet its quality-ofservice(QoS) requirements, if not cause widespread service outages and potentially total network breakdown [1], [2], [3], [4], [5], [6]. Robust network design and management strategies to deal with unexpected events must be in place to account for potential disruptions to network connectivity. A critical aspect of these strategies is their ability to pro-actively assess network vulnerability to disruptive events, in order to protect network elements ${ }^{1}$ against natural disaster and malicious human acts and mitigate the impact of such acts on network performance and survivability.

Over the last few years, a considerable amount of research effort has focused on developing approaches and methods to assess network vulnerability to disruptive events [1]. Central to these research studies is the use of graph theoretic measures to determine how vi-

- T. N. Dinh, Y. Xuan, M. T. Thai are with the Dept. of Comp. E Info. Sci. E Eng., University of Florida, Gainesville, FL, 32611.

E-mail: \{tdinh, yxuan, mythai\}@cise.ufl.edu.

- Panos M. Pardalos is with Industrial and System Engineering Dept., University of Florida at Gainesville, FL, 32611.

Email:pardalos@ufl.edu.

- T. Znati is with Computer Science Dept., University of Pittsburgh, Pittsburgh, PA 15215.

Email: znati@cs.pitt.edu.

1. In this paper, the term "network elements" is used to refer network nodes, communications links or a combination thereof. tal a network element is to the survivability of the network when faced with disruptive events. A number of these studies proposed global graph measures, such as cyclomatic number, maximum network circuits, $\alpha$ index, $\beta$-index and $\gamma$-index, as a single index to capture the network structure. The global index, derived from the basic properties of the graph, namely the number of vertices, edges and pairwise shortest paths, is then used to assess and compare the vulnerability of different networks. Although relatively straightforward to calculate, global measures can neither be rigorously mapped to the over network connectivity nor reveal the set of most critical vertices and edges. Such a shortcoming limits considerably their suitability for assessing network vulnerability. Other research studies proposed local nodal centrality [7], such as degree centrality, betweenness centrality and closeness centrality, as a local network measure for network vulnerability. These local measures, which emphasize the relative topological characteristics of individual edges and nodes within a network, are used to identify and quantify critical network elements of the network, and evaluate network vulnerability. Unfortunately, the inability of local network measures to capture global network connectivity raises concerns about their validity to accurately assess network vulnerability and reveal the global damage that may be inflicted on the network when subjected to failures and attacks.

In this paper, we propose a new framework for accurately assessing network vulnerability. The basic tenet of our approach is to model the network as a connected directed graph and explore the number of 
necessary disruptive events, in terms of connectivity failures between pairs of nodes, that can cause a specific level of network disruption. To this end, we quantify the connectivity of each node pair as 1 if the pair is strongly connected and as 0 otherwise. The pairwise connectivity quantification is then used to determine the minimized set of network elements whose removal from the graph incurs a specific level of network disruption. It is worth noting that the the pairwise connectivity measure correlates with the number of network elements to be removed for the network to incur a level of disruption. As such, the measure can be used not only to assess the vulnerability of the network against disruptive events, but equally important to evaluate the global damage different levels of disruptive events may cause.

Based on the concept of pairwise connectivity, network vulnerability assessment can be formulated as a graph-theoretical optimization problem: Find a minimized set of network elements whose removal causes a specific level of network pairwise degradation. The minimized set of network elements is thereafter referred to as $\beta$-disruptor, where $0 \leq \beta<1$ denotes the fraction of pairwise connectivity degradation that is caused by the failure of the minimized set elements. Two new optimization problems, namely $\beta$ vertex disruptor and $\beta$-edge disruptor, are introduced and proved to be NP-complete. We also propose two pseudo-approximation algorithms, with provable performance bounds, to these optimization problems, thereby ensuring the feasibility of the proposed network vulnerability assessment framework.

The advantage of the $\beta$-disruptor based framework, in comparison with node centrality based frameworks, can be briefly illustrated using Fig.1, where two strongly connected networks, $A$ and $B$, with 7 vertices each, are depicted. Using node degree centrality as a vulnerability measure, reveals that removing the black vertex with maximum outgoing degree 5 in Fig. 1-(a) leaves the network $A$ still strongly connected with 5 vertices; whereas, removing the black vertex with maximum outgoing degree 4 in Fig. 1-(b) partitions the graph into two strongly connected components. Therefore, the node-centrality based assessment leads to the conclusion that $A$ is less vulnerable than $B$. The analysis of the network vulnerability based on the proposed framework, however, shows that deleting only the grey vertex in $A$ is sufficient to decrease the overall network connectivity to 0 . Furthermore, the analysis shows that at least 3 vertices in $B$ must be removed to reduce the overall connectivity of the network to 0 . It is clear, therefore, that the $\beta$-disruptor framework leads to the more accurate conclusion that network $A$ is in fact more vulnerable than network $B$.

Another aspect of the proposed framework is the ability to explore different levels of disruption (different values of $\beta$ ), which can be used to gain deeper

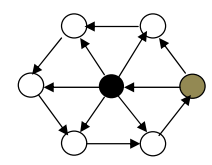

(a)

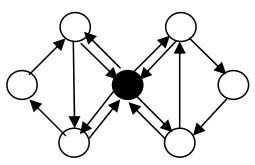

(b)
Fig. 1. After the "central" vertex (in black) with maximum out-going degree is removed, network (a) is still strongly connected while (b) is fragmented; however in fact, only removing one vertex (in grey) is enough to destroy network (a).

insight into network structure and robustness in various operating environments. Several recent studies in the context of wireless networks have focused on the impact of node and edge removal on network connectivity[8][9][10]. It is worth noting that most of these studies are not concerned with quantifying the level of network disconnectivity, a weaker objective than that of the $\beta$-disruptor framework. We argue that quantifying network connectivity is essential to assessing network vulnerability to disruptive events and evaluate the level of fragmentation these events may inflict on the network. A scale-free network, for example, can tolerate high random failure rates, as the failure of boundary vertices may not significantly reduce the network connectivity even though the entire graph becomes disconnected [11]. Furthermore, different disruption levels may be caused by different network elements. The node centrality based vulnerability assessment framework, for example, only identifies a set of nodes with non-increasing degrees with no consideration of the disruption level the removal of these sets of nodes may cause. The ability to quantify the disconnectivity level, a unique feature of $\beta$-disruptor framework, is valuable in assessing accurately network vulnerability to disruptive events and predicting the impact of these events on network operations.

The main contributions of this paper are as follows:

- A novel, pairwise-connectivity-centric framework for investigating vulnerability assessment as an optimization problem, referred to as $\beta$-disruptor, on general graphs. Two versions of the problem, namely $\beta$-vertex disruptor and $\beta$-edge disruptor, are considered;

- A proof of the NP-completeness of the two versions of the $\beta$-disruptor problem, including the proof that no polynomial-time approximation scheme (PTAS) exists for $\beta$-vertex disruptor;

- An $O\left(\log ^{\frac{3}{2}} n\right)$ pseudo-approximation algorithm for $\beta$-vertex disruptor and an $O(\log n \log \log n)$ pseudo-approximation algorithm for $\beta$-edge disruptor. These algorithms can be applied to both homogeneous networks and heterogeneous networks with unidirectional links and non-uniform nodal properties.

\subsection{Model and Definitions}

Besides the homogeneous network model consisting of uniform nodes and bidirectional links, the hetero- 
geneous network model, where various interacting elements of different kinds are connected through unidirectional links with non-uniform expenses, finds numerous applications nowadays [12], [13], [14], but as well, exhibits multiple difficulties for optimization and maintenance. In the light of this, we abstract our general network model as a directed graph $G(V, E)$, where $V$ refers to a set of nodes and $E$ refers to a set of unidirectional links. The expense of each directed edge $(u, v)$ between vertex $u$ and $v$ is quantified as a nonnegative value $c(u, v)$, for all the $m=|E|$ links among $n=|V|$ nodes. As mentioned above, our evaluation over the network vulnerability is based on the value of overall pairwise connectivity in the abstracted graph, which is defined as follows: given any vertex pair $(u, v) \in V \times V$ in the graph, we say that they are connected iff there exists paths between $u$ and $v$ in both directions in $G$, i.e., strongly connected to each other. The pairwise connectivity $p(u, v)$ is quantified as 1 if this pair is connected, 0 otherwise. Since the main purpose of network lies in connecting all the interacting elements, we study on the aggregate pairwise connectivity between all pairs, that is, the sum of quantified pairwise connectivity, which we denote as $\mathcal{P}(G)=\sum_{u, v \in V \times V} p(u, v)$ for graph $G$. Apparently $\mathcal{P}(G)$ is maximized at $\left(\begin{array}{l}n \\ 2\end{array}\right)$ when $G$ is a strongly connected graph. Based on this, we have:

Definition 1: (Edge disruptor) Given $0 \leq \beta \leq 1$, a subset $S \subset E$ in $G=(V, E)$ is a $\beta$-edge disruptor if the overall pairwise connectivity in the $G[E \backslash S]$, obtained by removing $S$ from $G$, is no more than $\beta\left(\begin{array}{l}n \\ 2\end{array}\right)$. By minimizing the cost of such edges in $S$, we have the $\beta$-edge disruptor problem, i.e., find a minimized $\beta$-edge disruptor in a strongly connected graph $G(V, E)$.

Similarly, we define $\beta$-vertex disruptor and its corresponding optimization problem:

$\beta$-vertex disruptor problem: Given a strongly connected graph $G(V, E)$ and a fixed number $0 \leq \beta \leq 1$, find a subset $S \subseteq V$ with the minimum size such that the total pairwise connectivity in $G_{[V \backslash S]}$, obtained by removing $S$ from $G$, is no more than $\beta\left(\begin{array}{c}n \\ 2\end{array}\right)$. Such a set $S$ is called $\beta$-vertex disruptor.

\subsection{Related Work}

Several research efforts have sought to address network vulnerability assessment using local network measures focused on the centrality of a vertex in the graph, including degree centrality, betweenness, closeness, and eigenvector centrality [7]. While these metrics can provide some insight into network vulnerability, they are are typically not accurate and in most cases fail to reveal the level of network disruption for different levels of attacks. Global graph measures have also been proposed to assess network vulnerability. These measures are mainly functions of graph properties, such as the number of vertices and edges, operational O-D pairs, operational paths, and minimum shortest paths [1], [2], [3]. Although useful in comparing different networks, the graph attributes, in some cases, cannot be calculated in polynomialtime for dense networks. Furthermore, the functions do not reveal the set of most critical vertices and edges in the network. As such, they may not be suitable to assess the network vulnerability, in terms of connectivity.

Several research studies, using concepts similar to our pairwise connectivity, have been recently investigated in [15], [16], [17], including average pairwise connectivity, pairwise connected ratio and cohesion. None of these studies, however, formulate network vulnerability as an optimization problem. Using an optimization based approach to network vulnerability enables a framework, where the hardness of the problem can be characterized and approximation algorithms with performance guarantees can be derived. Moreover, the problem $\beta$-disruptor studied in this paper take into account the roles of all edges and vertices in the global network connectivity, thereby providing the basis for a thorough analysis of the proposed vulnerability framework.

Finally, a number of research studies focused on a subproblem of the vulnerability assessment problem, namely the Critical Vertex/Edge, where the main objective is to determine the minimum number of vertices/edges whose removal disconnects the graph. Several heuristics were proposed to address this problem, including in the context of wireless networks [8][9][10]. The proposed heuristics, however, do not provide performance guarantees. Furthermore, the research studies in wireless environments focus on what elements of the graph cause the network to be disconnected, with no useful insight into the level of fragmentation caused by disruptive events of different scale and impact. As such, these approaches are limited in assessing network vulnerability accurately.

\section{Hardness Results}

In this section we show that both the $\beta$-edge disruptor and $\beta$-vertex disruptor in directed graph are NP-complete which thus have no polynomial time exact algorithms unless $\mathrm{P}=\mathrm{NP}$. We state a stronger result that both problems are NP-complete even in undirected graph with unit cost edges.

Note that only in this section we consider the problem for undirected graph $G(V, E)$. All results in other sections are studied on directed graphs, thus solving both homogeneous and heterogeneous networks.

\subsection{NP-completeness of $\beta$-edge disruptor}

We use a reduction from the balanced cut problem.

Definition 2: A cut $\langle S, V \backslash S\rangle$ corresponding to a subset $S \in V$ in $G$ is the set of edges with exactly one endpoint in $S$. The cost of a cut is the sum of its edges' costs (or simply its cardinality in the case all edges have unit costs). We often denote $V \backslash S$ by $\bar{S}$. 
$\mathrm{G}(\mathrm{V}, \mathrm{E})$

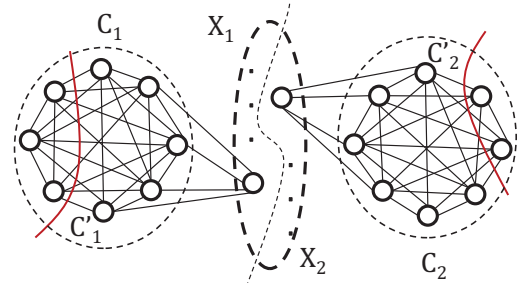

Fig. 2. Construction of $H\left(V_{H}, E_{H}\right)$ from $G(V, E)$

Finding a min cut in the graph is polynomial solvable [18]. However, if one asks for a somewhat "balanced" cut of minimum size, the problem becomes intractable. A balanced cut is defined as following:

Definition 3: (Balanced cut) An $f$-balanced cut of a graph $G(V, E)$, where $f: \mathbb{Z}^{+} \rightarrow \mathbb{R}^{+}$, asks us to find a cut $\langle S, \bar{S}\rangle$ with the minimum size such that $\min \{|S|,|\bar{S}|\} \geq f(|V|)$.

Abusing notations, for $0<c \leq \frac{1}{2}$, we also use $c$ balanced cut to find the cut $\langle S, \bar{S}\rangle$ with the minimum size such that $\min \{|S|,|\bar{S}|\} \geq c|V|$. We will use the following results on balanced cut shown in [19]:

Corollary 1: (Monotony) Let $g$ be a function with

$$
0 \leq g(n)-g(n-1) \leq 1
$$

Then $f(n) \leq g(n)$ for all $n$, implies $f$-balanced cut is polynomially reducible to $g$-balanced cut.

Corollary 2: (Upper bound) $\alpha n^{\epsilon}$-balanced cut is NPcomplete for $\alpha, \epsilon>0$.

It follows from Corollaries 1 and 2 that for every $f=$ $\Omega\left(\alpha n^{\epsilon}\right) f$-balanced cut is NP-complete. We are ready to prove the NP-completeness of $\beta$-edge disruptor:

Theorem 1: ( $\beta$-edge disruptor NP-completeness) $\beta$ edge disruptor in undirected graph is NP-complete even if all edges have unit weights.

Proof: We prove the result for the special case when $\beta=\frac{1}{2}$. For other values of $\beta$ the proof can go through with a slight modification of the reduction. We shall assume that $n$, the number of nodes is a sufficient large number (for our proof $n>10^{3}$ ).

Consider the decision version of the problem

$$
\frac{1}{2} \text {-ED }=\left\{\langle G, K\rangle \mid G \text { has a } \frac{1}{2} \text {-edge disruptor of size } K\right\}
$$

To show that $\frac{1}{2}$-ED is in NP-complete we must show that it is in NP and that all NP-problems are polynomial time reducible to it. The first part is easy; given a candidate subset of edges, we can easily check in polynomial time if it is a $\beta$-edge disruptor of size $K$. To prove the second part, we show that $f$-balanced cut is polynomial time reducible to $\frac{1}{2}$-ED where $f=\left\lfloor\frac{n-\sqrt{2\left\lfloor\frac{n^{2}}{3}\right\rfloor+n}}{2}\right\rfloor$.

Let $G(V, E)$ be a graph in which one seeks to find a $f$-balanced cut of size $k$. Construct the following graph $H\left(V_{H}, E_{H}\right): V_{H}=V \cup C_{1} \cup C_{2}$ where $C_{1}, C_{2}$ are two cliques of size $\left\lfloor\frac{n^{2}}{3}\right\rfloor$. Denote by $N=\left|V_{H}\right|=$ $2\left\lfloor\frac{n^{2}}{3}\right\rfloor+n$ the total number of nodes in $H$. In addition to edges in $G, C_{1}$, and $C_{2}$, connect each vertex $v \in V$ to $\left\lfloor\frac{n^{2}}{4}\right\rfloor+1$ vertices in $C_{1}$ and $\left\lfloor\frac{n^{2}}{4}\right\rfloor+1$ vertices in $C_{2}$ so that degree difference of nodes in the cliques are at most one. We illustrate the construction of $H\left(V_{H}, E_{H}\right)$ in Figure 2.

We show that there is a $f$-balanced cut of size $k$ in $G$ iff $H$ has an $\frac{1}{2}$-edge disruptor of size $K=$ $n\left(\left\lfloor\frac{n^{2}}{4}\right\rfloor+1\right)+k$ where $0 \leq k \leq\left\lfloor\frac{n^{2}}{4}\right\rfloor$. Note that the cost of any cut $\langle S, V \backslash S\rangle$ in $G$ is at most $|S||V \backslash S| \leq$ $\left\lfloor\frac{(|S|+|V \backslash S|)^{2}}{4}\right\rfloor=\left\lfloor\frac{n^{2}}{4}\right\rfloor$.

On one hand, an $f$-balanced cut $\langle S, \bar{S}\rangle$ of size $k$ in $G$ induces a cut $\left\langle C_{1} \cup S, C_{2} \cup \bar{S}\right\rangle$ with size exactly $n\left(\left\lfloor\frac{n^{2}}{4}\right\rfloor+1\right)+k$. If we select the cut as the disruptor, the pairwise connectivity will be at most $\frac{1}{2}\left(\begin{array}{c}N \\ 2\end{array}\right)$.

On the other hand, assume that $H$ has an $\frac{1}{2}$-edge disruptor of size $K=n\left(\left\lfloor\frac{n^{2}}{4}\right\rfloor+1\right)+k$. Remove the edges in the disruptor to reduce the pairwise connectivity to at most $\frac{1}{2}\left(\begin{array}{c}N \\ 2\end{array}\right)$. Since cutting $n$ nodes in $C_{1}$ or $C_{2}$ from the cliques requires removing at least $n\left(\left\lfloor\frac{n^{2}}{3}\right\rfloor-n\right)>n\left(\left\lfloor\frac{n^{2}}{4}\right\rfloor+1\right)+k$ edges, let $C_{1}^{\prime} \subseteq C_{1}$ and $C_{2}^{\prime} \subseteq C_{2}$ be giant connected subsets that induce connected subgraphs in $C_{1}$ and $C_{2}$. These subsets must satisfy $\left|C_{1}^{\prime}\right|+\left|C_{2}^{\prime}\right|>\left|C_{1}\right|+\left|C_{2}\right|-n$. Denote by $X_{1}, X_{2}$ the subsets of nodes in $V$ that are connected to $C_{1}^{\prime}$ and $C_{2}^{\prime}$ respectively. We have $X_{1} \cap X_{2}=\emptyset$ otherwise $C_{1}^{\prime}$ and $C_{2}^{\prime}$ will be connected; then, the pairwise connectivity will exceed $\frac{1}{2}\left(\begin{array}{c}N \\ 2\end{array}\right)$.

We will modify the disruptor without increasing its size and the pairwise connectivity such that no nodes in the the cliques are cut off i.e. we alter the disruptor until $C_{1}^{\prime}=C_{1}$ and $C_{2}^{\prime}=C_{2}$. For each $u \in C_{1} \backslash C_{1}^{\prime}$ remove from the disruptor all edges connecting $u$ to $C_{1}^{\prime}$ and add to the disruptor all edges connecting $u$ to $X_{2}$. This will attach $u$ to $C_{1}^{\prime}$ while reducing the size of the disruptor at least $\left(\left\lfloor\frac{n^{2}}{3}\right\rfloor-n\right)-n$. At the same time select an arbitrary node $v \in X_{1}$ and add to the disruptor all remaining $v^{\prime}$ s adjacent edges. This increases the size of the disruptor at most $\left(\left\lfloor\frac{n^{2}}{4}\right\rfloor+1\right)+n$ while making $v$ isolated. By doing so we decrease the size of the disruptor by $\left(\left\lfloor\frac{n^{2}}{3}\right\rfloor-n\right)-n-\left(\left(\left\lfloor\frac{n^{2}}{4}\right\rfloor+1\right)+\right.$ $n)>0$. In addition, the pairwise connectivity will not increase as we connect $u$ to $C_{1}^{\prime}$ and at the same time disconnect $v$ from $C_{1}^{\prime}$.

If $X_{1}=\emptyset$, we can select $v \in X_{2}$ as in that case $\left|C_{2}^{\prime} \cup X_{2}\right|>\left|C_{1}^{\prime} \cup X_{1}\right|$ that makes sure the pairwise connectivity will not increase. We repeat the same process for every node in $C_{2} \backslash C_{2}^{\prime}$. Since $\left|\left(C_{1} \backslash C_{1}^{\prime}\right) \cup\left(C_{2} \backslash C_{2}^{\prime}\right)\right|<n$, the whole process finishes in less than $n$ steps and results in $C_{1}^{\prime}=C_{1}$ and $C_{2}^{\prime}=C_{2}$.

We will prove that $X_{1} \cup X_{2}=V$ i.e. $\left\langle X_{1}, X_{2}\right\rangle$ induces a cut in $G$. Assume not, the cost to separate $C_{1} \cup X_{1}$ from $C_{2} \cup X_{2}$ will be at least $\left(\left\lfloor\frac{n^{2}}{4}\right\rfloor+1\right)\left(\left|V-X_{1}\right|+\mid V-\right.$ $\left.X_{2} \mid\right)=\left(\left\lfloor\frac{n^{2}}{4}\right\rfloor+1\right)\left(2 n-\left|X_{1}\right|-\left|X_{2}\right|\right) \geq\left(\left\lfloor\frac{n^{2}}{4}\right\rfloor+1\right)(n+1)>$ $n\left(\left\lfloor\frac{n^{2}}{4}\right\rfloor+1\right)+k$ that is a contradiction.

Since $X_{1} \cup X_{2}=V$ we have that the disruptor induces a cut in $G$. To have the pairwise connectivity 
at most $\frac{1}{2}\left(\begin{array}{c}N \\ 2\end{array}\right)$ both $\left(C_{1} \cup X_{1}\right)$ and $\left(C_{2} \cup X_{2}\right)$ must have size at least $\frac{N-\sqrt{N}}{2}$. If follows that $X_{1}$ and $X_{2}$ must have size at least $f(n)=\left\lfloor\frac{n-\sqrt{2\left\lfloor\frac{n^{2}}{3}\right\rfloor+n}}{2}\right\rfloor$. The cost of the cut induced by $\left\langle X_{1}, X_{2}\right\rangle$ in $G$ will be $n\left(\left\lfloor\frac{n^{2}}{4}\right\rfloor+1\right)+k-n\left(\left\lfloor\frac{n^{2}}{4}\right\rfloor+1\right)=k$.

\subsection{Hardness of $\beta$-vertex disruptor}

Theorem 2: $\beta$-vertex disruptor in undirected graph is NP-complete.

Proof: We present a polynomial-time reduction from Vertex Cover (VC), an NP-hard problem [20]:

Instance: Given a graph $G$ and a positive integer $k$.

Question: Does $G$ have a VC of size at most $k$ ?

to a decision version of $\beta$-vertex disruptor when $\beta=0$

Instance: Given a graph $G$ and a positive integer $k$

Question: Does $G$ have a $\beta$-vertex disruptor of size at most $k$ when $\beta=0$ ?

Pairwise connectivity equals zeros if and only if the complement set of the disruptor is an independent set or in other words the disruptor must be a VC.

Theorem 3: Unless $\mathrm{P}=\mathrm{NP}, \beta$-vertex disruptor cannot be approximated within a factor of 1.36 .

Proof: We use the same reduction in Theorem 2. Assume that we can approximate $\beta$-vertex disruptor within a factor less than 1.36 when $\beta=0$. In [21], Dinur and Safra showed that approximating VC within constant factor less than 1.36 is NP-hard. Since we have an one-to-one mapping between the set of vertex disruptors when $\beta=0$ and the set of VCs, it follows that we can approximate VC within a factor less than 1.36 (contradiction).

\section{ApProximating $\beta$-EDGe DisRuptor USING TREE DECOMPOSITION}

In this section, we present an $O\left(\log ^{\frac{3}{2}} n\right)$ pseudoapproximation algorithm for the $\beta$-edge disruptor problem in the case when all edges have uniform cost i.e. $c(u, v)=1 \forall(u, v) \in E(G)$. Formally, our algorithm finds in a uniform directed graph $G$ a $\beta^{\prime}$-edge disruptor whose the cost is at most $O\left(\log ^{\frac{3}{2}} n\right) \mathrm{OPT}_{\beta-E D}$, where $\frac{\beta^{\prime}}{4}<\beta<\beta^{\prime}$ and $\mathrm{OPT}_{\beta-E D}$ is the cost of an optimal $\beta$-edge disruptor.

As shown in Algorithm 1, the proposal algorithm consists of two main steps. First, we constructs a decomposition tree of $G$ by recursively partitioning the graph into two halves with directed $c$-balanced cut. Second, we solve the problem on the obtained tree using a dynamic programming algorithm and transfer this solution to the original graph. These two main steps are explained in the next two sections.

\subsection{Balanced Tree-Decomposition}

A tree decomposition of a graph is a recursive partitioning of the node set into smaller and smaller pieces until each piece contains only one single node. We show the tree construction in Algorithm 1 (line 1 to

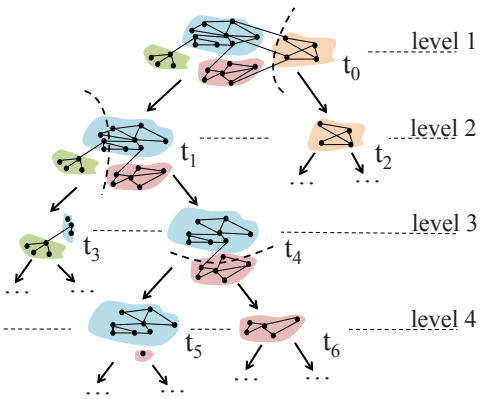

Fig. 3. A part of a decomposition tree. $F=\left\{t_{2}, t_{3}, t_{5}, t_{6}\right\}$ is a $G$-partitionable. The corresponding partition $\left\{V\left(t_{2}\right), V\left(t_{3}\right), V\left(t_{5}\right), V\left(t_{6}\right)\right\}$ in $G$ can be obtained by using cuts at ancestors of nodes in $F$ i.e. $t_{0}, t_{1}, t_{4}$.

11). Our decomposition tree is a rooted binary tree whose leaves represent nodes in $G$.

Definition 4: Given a directed graph $G(V, E)$ and a subset of vertices $S \subset V$. We denote the set of edges outgoing from $S$ by $\delta^{+}(S)$; the set of edges incoming to $S$ by $\delta^{-}(S)$. A cut $(S, V \backslash S)$ in $G$ is defined as $\delta^{+}(S)$. A $c$-balanced cut is a cut $(S, V \backslash S)$ s.t. $\min \{|S|, \mid V \backslash$ $S \mid\} \geq c|V|$. The directed $c$-balanced cut problem is to find the minimum $c$-balanced cut.

Note that a cut $(S, V \backslash S)$ separate pairs $(u, v) \in$ $S \times(V \backslash S)$ as paths from $v$ to $u$ cannot exist i.e. no SCC can contain vertex in both $S$ and $V \backslash S$.

The decomposition procedure is as follows. We start with the tree $T$ containing only one root node $t_{0}$. We associate the root node $t_{0}$ with the vertex set $V$ of $G$ i.e. $V\left(t_{0}\right)=V(G)$. For each node $t_{i} \in T$ whose $V\left(t_{i}\right)$ contains more than one vertex and $V\left(t_{i}\right)$ has not been partitioned, we partition the subgraph $G_{\left[V\left(t_{i}\right)\right]}$ induced by $V\left(t_{i}\right)$ in $G$ using a $c$-balanced cut algorithm. In detail, we use the directed $c$-balanced cut algorithm presented in [22] that finds in polynomial time a $c^{\prime}$-balanced cut within a factor of $O(\sqrt{\log n})$ from the optimal $c$-balanced cut for $c^{\prime}=\alpha c$ and fixed constant $\alpha$. The constant $c$ is chosen to be $1-\sqrt{\frac{\beta}{\beta^{\prime}}}$. Create two child nodes $t_{i 1}, t_{i 2}$ of $t_{i}$ in $T$ corresponding to two sets of vertices of $G_{\left[V\left(t_{i}\right)\right]}$ separated by the cut. We associate with $t_{i}$ a cut cost $\operatorname{cost}\left(t_{i}\right)$ equal to the cost of the $c$-balanced cut.

We define the root node $t_{0}$ to be on level 1 . If a node is on level $l$, all its children are defined to be on level $l+1$. Note that collections of subsets of vertices in $G$ that correspond to nodes in a same level of $T$ induces a partition in $G$.

One important parameter of the decomposition tree is the height i.e. the maximum level of nodes in $T$. Using balanced cuts guarantees a small height of the tree that in turn leads to a small approximation ratio. When separating $V\left(t_{i}\right)$ using the balanced cut, the size of the larger part is at most $\left(1-c^{\prime}\right)\left|V\left(t_{i}\right)\right|$. Hence, we can prove by induction that if a node $t_{i}$ is on level $k$, the size of the corresponding collection $V\left(t_{i}\right)$ is at most $|V| \times\left(1-c^{\prime}\right)^{k-1}$. It follows that the tree's height is at most $O\left(-\log _{1-c^{\prime}} n\right)=O(\log n)$. 
Algorithm 1. $\beta$-edge Disruptor

Input: Uniform edges' weight directed graph $G=(V, E)$ and $0 \leq \beta<\beta^{\prime}<1$

Output: A $\beta^{\prime}$-edge disruptor of $G$.

$/ *$ Construct the decomposition tree */

1. $c \leftarrow 1-\sqrt{\frac{\beta}{\beta^{\prime}}}$.

2. $T\left(V_{T}, E_{T}\right) \leftarrow\left(\left\{t_{0}\right\}, \phi\right), V\left(t_{0}\right) \leftarrow V(G), l\left(t_{0}\right)=1$

3. while $\exists$ unvisited $t_{i}$ with $\left|V\left(t_{i}\right)\right| \geq 2$ do

4. Mark $t_{i}$ visited, create new child nodes $t_{i 1}, t_{i 2}$ of $t_{i}$.

5. $V_{T} \leftarrow V_{T} \cup\left\{t_{i 1}, t_{i 2}\right\}$

6. $E_{T} \leftarrow E_{T} \cup\left\{\left(t_{i}, t_{i 1}\right),\left(t_{i}, t_{i 2}\right)\right\}$

7. Separate $G_{\left[V\left(t_{i}\right)\right]}$ using directed $c$-balanced cut.

8. Associate $V\left(t_{i 1}\right), V\left(t_{i 2}\right)$ with two separated components.

9. $\operatorname{cost}\left(t_{i}\right) \leftarrow$ The cost of the balanced cut

$/{ }^{*}$ Find the minimum cost $G$-partitionable */

10. Traverse $T$ in post-order, for each $t_{i} \in T$ do

11. for $p \leftarrow 0$ to $\beta^{\prime}\left(\begin{array}{l}n \\ 2\end{array}\right)$

12. $\quad$ if $\mathcal{P}\left(G_{\left[V\left(t_{i}\right)\right]}\right) \leq p$ then $\operatorname{cost}\left(t_{i}, p\right) \leftarrow 0$

13. $\quad$ else $\operatorname{cost}\left(t_{i}, p\right) \leftarrow \min \left\{\operatorname{cost}\left(t_{i 1}, p_{1}\right)+\right.$ $\left.\operatorname{cost}\left(t_{i 2}, p_{2}\right)+\operatorname{cost}\left(t_{i}\right) \mid p_{1}+p_{2}=p\right\}$

14. Find $F_{\beta^{\prime}}^{\mathrm{opt}}$ associating with $T_{\beta^{\prime}}^{\mathrm{opt}}=\min _{p \leq \beta^{\prime}\left(\begin{array}{c}n \\ 2\end{array}\right)}\left\{\cos t\left(t_{0}, p\right)\right\}$ 15. Return union of $c$-balanced cuts at $t_{i} \in \mathcal{A}\left(F_{\beta^{\prime}}^{\mathrm{opt}}\right)$.

\subsection{Algorithm}

In this section, we present the second main step which uses the dynamic programming to search for the right set of nodes in $T$ that induces an cost-efficient partition in $G$ whose pairwise connectivity is at most $\beta^{\prime}\left(\begin{array}{l}n \\ 2\end{array}\right)$. The details of this step are shown in Algorithm 1 (lines 12 to 18 ).

Denote a set $F=\left\{t_{u_{1}}, t_{u_{2}}, \ldots, t_{u_{k}}\right\} \subset V_{T}$ where $V_{T}$ is the set of vertices in $T$ so that $V\left(t_{u_{1}}\right), V\left(t_{u_{2}}\right), \ldots, V\left(t_{u_{k}}\right)$ is a partition of $V(G)$ i.e. $V(G)=\biguplus_{h=1} V_{u_{h}}$. We say such a subset $F$ is $G$ partitionable. Denote by $\mathcal{A}\left(t_{i}\right)$ the set of ancestors of $t_{i}$ in $T$ and $\mathcal{A}(F)=\bigcup_{t_{i} \in F} \mathcal{A}\left(t_{i}\right)$. It is clear that a $F$ is $G$-partitionable if and only if $F$ satisfies:

1) $\forall t_{i}, t_{j} \in F: t_{i} \notin \mathcal{A}\left(t_{j}\right)$ and $t_{j} \notin \mathcal{A}\left(t_{i}\right)$

2) $\forall t_{i} \in V_{T}, t_{i}$ is a leaf: $\mathcal{A}\left(t_{i}\right) \cap F \neq \phi$

In case $F$ is $G$-partitionable, we can separate $V\left(t_{u_{1}}\right), V\left(t_{u_{2}}\right), \ldots, V\left(t_{u_{k}}\right)$ in $G$ by performing the cuts corresponding to ancestors of node in $F$ during the tree construction. For example in Figure 3 , we show a decomposition tree with a $G$ partitionable set $\left\{t_{2}, t_{3}, t_{5}, t_{6}\right\}$. The corresponding partition $\left\{V\left(t_{2}\right), V\left(t_{3}\right), V\left(t_{5}\right), V\left(t_{6}\right)\right\}$ in $G$ can be obtained by cutting $V\left(t_{0}\right), V\left(t_{1}\right), V\left(t_{4}\right)$ successively using balanced cuts in the tree construction. The cut cost, hence, will be $\operatorname{cost}\left(t_{0}\right)+\operatorname{cost}\left(t_{1}\right)+\cos t\left(t_{4}\right)$. In general, the total cost of all the cuts to separate $V\left(t_{u_{1}}\right), V\left(t_{u_{2}}\right), \ldots, V\left(t_{u_{k}}\right)$ will be $\operatorname{cost}(F)=$ $\sum_{t_{u} \in \mathcal{A}(F)} \operatorname{cost}\left(t_{u}\right)$. The pairwise connectivity of $G$ is then $\mathcal{P}(F)=\sum_{t_{u} \in F} \mathcal{P}\left(G_{\left[V\left(t_{u}\right)\right]}\right)$. We wish to find $F$ so that $\mathcal{P}(F) \leq \beta^{\prime}\left(\begin{array}{l}n \\ 2\end{array}\right)$ i.e. the union of cuts to separate $V\left(t_{u_{1}}\right), V\left(t_{u_{2}}\right), \ldots, V\left(t_{u_{k}}\right)$ forms a $\beta^{\prime}$-edge disruptor in $G$. Because of the suboptimal structure in $T$, finding such a $G$-partitionable subset $F$ in $V_{T}$ with the minimum $\operatorname{cost}(F)$ can be done in $O\left(n^{3}\right)$ using dynamic programming.

Denote $\operatorname{cost}\left(t_{i}, p\right)$ the minimum cut cost to make the pairwise connectivity in $G_{\left[V\left(t_{i}\right)\right]}$ equal to $p$ using only cuts corresponding to nodes in the subtree rooted at $t_{i}$. The minimum cost for a $G$-partitionable subset $F$ that induces a $\beta^{\prime}$-edge disruptor of $G$ is then

$T_{\beta^{\prime}}^{\text {opt }}=\min _{p \leq \beta^{\prime}\left(\begin{array}{l}n \\ 2\end{array}\right)}\left\{\operatorname{cost}\left(t_{0}, p\right)\right\}$, where $t_{0}$ is the root node in $T$.

We can easily derive the recursive formula:

$\operatorname{cost}\left(t_{i}, p\right)=\left\{\begin{array}{l}0 \quad \text { if } \mathcal{P}\left(G_{\left[V\left(t_{i}\right)\right]}\right) \leq p \\ \min _{\pi \leq p} \operatorname{cost}\left(t_{i 1}, \pi\right)+\operatorname{cost}\left(t_{i 2}, p-\pi\right)+\operatorname{cost}\left(t_{i}\right) \text { if not }\end{array}\right.$ where $t_{i 1}, t_{i 2}$ are children of $t_{i}$.

In the first case, when $\mathcal{P}\left(G_{\left[V\left(t_{i}\right)\right]}\right) \leq p$ we cut no edges in $G_{\left[V\left(t_{i}\right)\right]}$ hence, $\operatorname{cost}\left(t_{i}, p\right)=0$. Otherwise, we try all possible combinations of pairwise connectivity $\pi$ in $V\left(t_{i 1}\right)$ and $p-\pi$ in $V\left(t_{i 2}\right)$. The combination with the smallest cut cost is then selected.

We now prove that $T_{\beta^{\prime}}^{\text {opt }} \leq O\left(\log ^{\frac{3}{2}} n\right) \mathrm{Opt}_{\beta-\mathrm{ED}}$, where $\mathrm{Opt}_{\beta}$-ED denotes the cost of the optimal $\beta$-edge disruptor in $G$.

Lemma 1: There exists a $G$-partitionable subset of $T$ that induces a $\beta^{\prime}$-edge disruptor whose cost is at most $O\left(\log ^{\frac{3}{2}} n\right) \mathrm{Opt}_{\beta \text {-ED }}$.

Proof: Let $D_{\beta}$ be an optimal $\beta$-edge disruptor in $G$ of size Opt ${ }_{\beta}$-ED and $\mathcal{C}_{\beta}=\left\{C_{1}, C_{2}, \ldots, C_{k}\right\}$ be the set of SCCs, after removing $D_{\beta}$ from $G$.

We construct a $G$-partitionable subset $X_{T}$ as in the Algorithm 2. We traverse tree $T$ in preorder i.e. every parent will be visited before its children. For each node $t_{i}$, we select $t_{i}$ into $X_{T}$ if there exists some component $C_{j} \in \mathcal{C}_{\beta}$ that $\left|V\left(t_{i}\right) \cap C_{j}\right| \geq(1-c)\left|V\left(t_{i}\right)\right|$ and no ancestors of $t_{i}$ have been selected into $X_{T}$. We can verify that $X_{T}$ satisfies two mentioned conditions of a $G$-partitionable subset. For each $C_{j} \in \mathcal{C}_{\beta}$, define

$$
N\left(C_{j}\right)=\left\{t_{i} \in T:\left|V\left(t_{i}\right) \cap C_{j}\right| \geq(1-c)\left|V\left(t_{i}\right)\right|\right\} .
$$

Since $V\left(t_{i}\right), t_{i} \in T$ are disjoint subsets. We have

$$
\begin{aligned}
\mathcal{P}\left(X_{T}\right) & \leq \sum_{t_{i} \in X_{T}}\left(\begin{array}{c}
\left|V\left(t_{i}\right)\right| \\
2
\end{array}\right) \\
& =\frac{1}{2} \sum_{C_{j} \in \mathcal{C}_{\beta}} \sum_{t_{i} \in N\left(C_{j}\right)}\left|V\left(t_{i}\right)\right|^{2}-\frac{n}{2} \\
& \leq \frac{1}{2} \sum_{C_{j} \in \mathcal{C}_{\beta}}\left(\sum_{t_{i} \in N\left(C_{j}\right)}\left|V\left(t_{i}\right)\right|\right)^{2}-\frac{n}{2} \\
& \leq \frac{1}{2} \sum_{C_{j} \in \mathcal{C}_{\beta}}\left(\sqrt{\beta^{\prime} / \beta}\left|C_{j}\right|\right)^{2}-\frac{n}{2} \\
& <\frac{\beta^{\prime}}{\beta} \frac{1}{2}\left(\sum_{C_{j} \in \mathcal{C}_{\beta}}\left|C_{j}\right|^{2}-n\right) \leq \beta^{\prime}\left(\begin{array}{l}
n \\
2
\end{array}\right)
\end{aligned}
$$




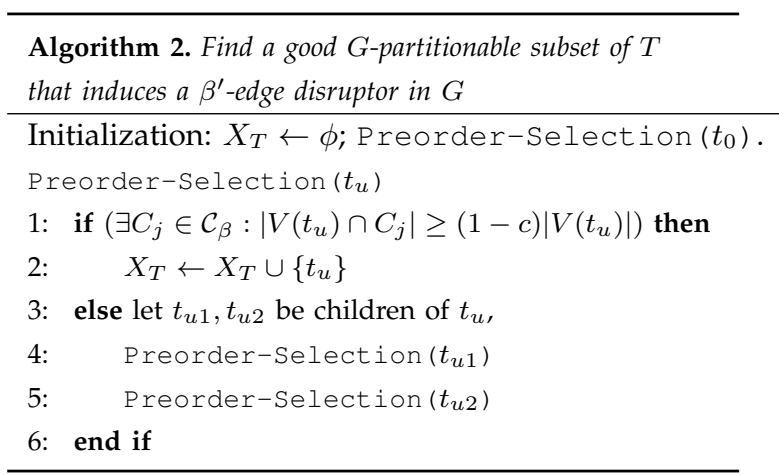

Finally we show that $\operatorname{cost}\left(X_{T}\right) \leq O\left(\log ^{\frac{3}{2}} n\right) \mathrm{Opt}_{\beta}$-ED. Let denote by $h(T)$ the height of $T$ and $L_{T}^{i}$ the set of nodes at the $i$ th level in $T_{G}$. We have:

$$
\operatorname{cost}\left(X_{T}\right)=\sum_{i=1}^{h(T)} \sum_{t_{u} \in\left(L_{T}^{i} \cap \mathcal{A}\left(X_{T}\right)\right)} \operatorname{cost}\left(t_{u}\right)
$$

If $t_{u} \in \mathcal{A}\left(X_{T}\right)$ then $t_{u}$ is not selected to $X_{T}$. Hence, there exists $C_{j} \in \mathcal{C}$ so that $\left|V\left(t_{u}\right) \cap C_{j}\right|<(1-c)\left|V\left(t_{u}\right)\right|$ (otherwise $t_{u}$ was selected into $X_{T}$ as it satisfied the conditions in the line 3, Algorithm 2). To guarantee $c<1-c$, we need $c<1 / 2$ i.e. $\beta>\frac{\beta^{\prime}}{4}$.

Since the edges in $D_{\beta}$ separate $C_{j}$ from the other SCCs, they also separates $C_{j} \cap V\left(t_{u}\right)$ from $V\left(t_{u}\right) \backslash C_{j}$ in $G_{\left[V\left(t_{u}\right)\right]}$. Denote by $\delta\left(t_{u}, D_{\beta}\right)$ the set of edges in $D_{\beta}$ separating $C_{j} \cap V\left(t_{u}\right)$ from $V\left(t_{u}\right) \backslash C_{j}$ in $G_{\left[V\left(t_{u}\right)\right]}$. Obviously, $\delta\left(t_{u}, D_{\beta}\right)$ is a directed $c$-balanced cut of $G_{\left[V\left(t_{u}\right)\right]}$. Since, the cut we used in the tree construction is only $O(\sqrt{\log n})$ times the optimal $c$-balanced cut. We have $\operatorname{cost}\left(t_{u}\right) \leq O(\sqrt{\log n})\left|\delta\left(t_{u}, D_{\beta}\right)\right|$.

Recall that if two nodes $t_{u}, t_{v}$ are on a same level then $V\left(t_{u}\right)$ and $V\left(t_{v}\right)$ are disjoint subsets. It follows that $\delta\left(t_{u}, D_{\beta}\right)$ and $\delta\left(t_{v}, D_{\beta}\right)$ are also disjoint sets. Therefore, the cut cost at the $i$ th level

$$
\begin{aligned}
& \sum_{t_{u} \in\left(L_{T}^{i} \cap \mathcal{A}\left(X_{T}\right)\right)} \operatorname{cost}\left(t_{u}\right) \\
\leq & O(\sqrt{\log n}) \sum_{t_{u} \in\left(L_{T}^{i} \cap \mathcal{A}\left(X_{T}\right)\right)}\left|\delta\left(t_{u}, D_{\beta}\right)\right| \\
\leq & O(\sqrt{\log n})\left|\bigcup_{t_{u} \in\left(L_{T}^{i} \cap \mathcal{A}\left(X_{T}\right)\right)} \delta\left(t_{u}, D_{\beta}\right)\right| \\
= & O(\sqrt{\log n}) \operatorname{Opt}_{\beta} \text {-ED }
\end{aligned}
$$

Since the number of levels $h(T)=O(\log n)$, by Eq. 1 we have $\operatorname{cost}\left(X_{T}\right) \leq O\left(\log ^{\frac{3}{2}} n\right) \operatorname{Opt}_{\beta}$-ED.

Since there exists a $G$-partitionable subset of $T$ that induces a $\beta^{\prime}$-edge disruptor whose cost is no more than $O\left(\log ^{\frac{3}{2}} n\right) \operatorname{Opt}_{\beta}$-ED as shown in Lemma 1 and the dynamic programming always finds the best latent solution in $T$, the following theorem follows.

Theorem 4: Algorithm 1 achieves a pseudoapproximation ratio of $O\left(\log ^{\frac{3}{2}} n\right)$ for the $\beta$-edges disruptor problem.

Time complexity: Construction of the decomposition tree takes $O\left(n^{9.5}\right)$. The major portion of time is for solving an semidefinite programming with $\Omega\left(n^{3}\right)$ constraints. Finding the optimal solution using Dynamic Programming takes $O\left(n^{5}\right)$. Hence, the overall time complexity is $O\left(n^{9.5}\right)$.

\section{$4 \beta$-VERTEX DISRUPTOR}

We present a polynomial time algorithm (Algorithm 3) that finds a $\beta^{\prime}$-vertex disruptor in the directed graph $G(V, E)$ whose the size is at most $O(\log n \log \log n)$ times the optimal $\beta$-vertex disruptor where $0<\beta<\beta^{\prime 2}$. The algorithm involves in two phases. In the first phase, we split each vertex $v \in V$ into two vertices $v^{+}$and $v^{-}$while putting an edge from $v^{-}$to $v^{+}$and show that removing $v$ in $G$ has the same effects as removing edge $\left(v^{+} \rightarrow v^{-}\right)$in the new graph. In the second phase, we try to decompose the new graph into SCCs capping the sizes of the largest component while minimizing the number of removed edges. We relax the constraints on the size of each component until the set of cut edges induces a $\beta^{\prime}$-vertex disruptor in the original graph $G$.

Given a directed graph $G(V, E)$ for which we want to find a small $\beta^{\prime}$-vertex disruptor, we split each vertex in $G$ into two new vertices to obtain a new directed graph $G^{\prime}\left(V^{\prime}, E^{\prime}\right)$ where

$$
\begin{aligned}
V^{\prime}= & \left\{v^{-}, v^{+} \mid v \in V\right\} \\
E^{\prime}= & \left\{\left(v^{-} \rightarrow v^{+}\right) \mid v \in V\right\} \\
& \cup\left\{\left(u^{+} \rightarrow v^{-}\right) \mid(u \rightarrow v) \in E\right\}
\end{aligned}
$$

The new graph $G^{\prime}\left(V^{\prime}, E^{\prime}\right)$ will have twice the number of vertices in $G$ i.e. $\left|V^{\prime}\right|=2|V|=2 n$. An example for the first phase is shown in Figure 4.

We set the costs of all edges in $E_{V}^{\prime}=\left\{\left(v^{-} \rightarrow\right.\right.$ $\left.\left.v^{+}\right) \mid v \in V\right\}$ to 1 and other edges in $E^{\prime}$ to $+\infty$ so that only edges in $E_{V}^{\prime}$ can be selected in an edge disruptor set. In implementation, it is safe to set the costs of edges not in $E_{V}^{\prime}$ to $O(n)$ noting that by paying a cost of $2 n$ we can effectively disconnect all edges in $E_{V}^{\prime}$.

Consider a directed edge disruptor set $D_{e}^{\prime} \subset E^{\prime}$ that contains only edge in $E_{V}^{\prime}$. We have a one-to-one correspondence between $D_{e}^{\prime}$ to a set $D_{v}=\left\{v \mid\left(v^{-} \rightarrow\right.\right.$ $\left.\left.v^{+}\right) \in D_{e}^{\prime}\right\}$ in $G(V, E)$ which is a vertex disruptor set in $G$. Since $G$ and $G^{\prime}$ have different maximum pairwise connectivity, $\frac{(n-1) n}{2}$ for $G$ and $\frac{(2 n-1) 2 n}{2}$ for $G^{\prime}$, the fractions of pairwise connectivity remaining in $G$ and $G^{\prime}$ after removing $D_{v}$ and $D_{e}^{\prime}$ are, however, not exactly equal to each other.

In the second phase of Algorithm 3, when separating a graph into SCCs, the smaller the sizes of SCCs, the smaller pairwise connectivity in the graph. However, the smaller the maximum size of each SCC, the more edges to be cut. We perform binary search to find a right upper bound for size of each SCC in $G^{\prime}$. In the algorithm, the lower bound and upper bound of the size of each SCC are denoted by $\beta\left|V^{\prime}\right|$ and $\bar{\beta}\left|V^{\prime}\right|$ respectively. At each step we try to find a minimum capacity edge set in $G^{\prime}\left(V^{\prime}, E^{\prime}\right)$ whose removal partitions the graph into strongly connected components of 


\section{Algorithm 3. $\beta^{\prime}$-vertex disruptor}

Input: Directed graph $G=(V, E)$ and fixed $0<\beta^{\prime}<1$.

Output: A $\beta^{\prime}$-vertex disruptor of $G$

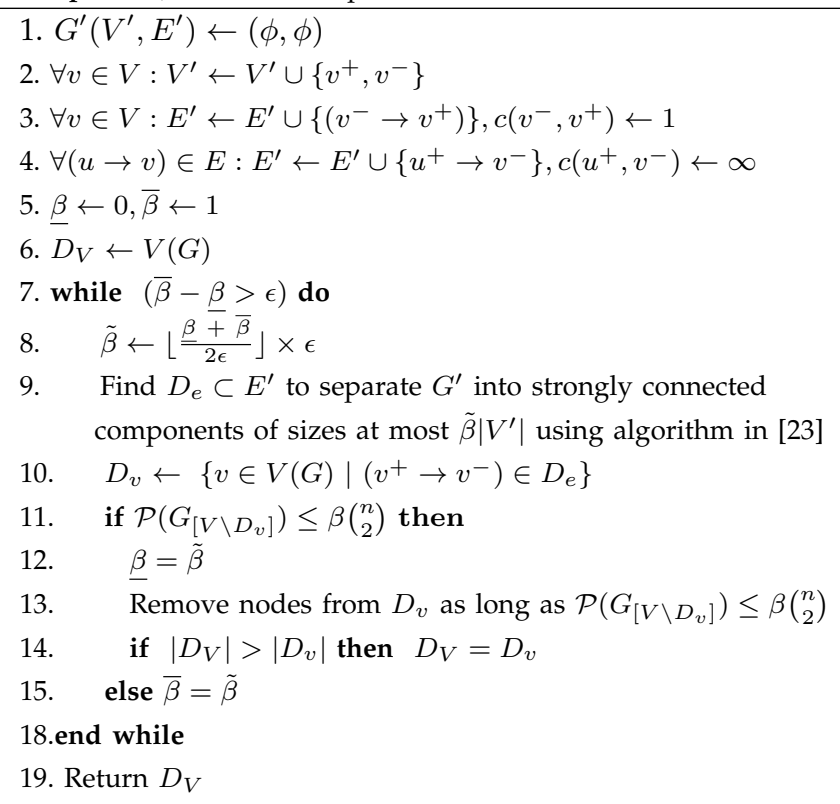

size at most $\tilde{\beta}\left|V^{\prime}\right|$, where $\tilde{\beta}=\left\lfloor\frac{\underline{\underline{\beta}}+\bar{\beta}}{2 \epsilon}\right\rfloor \times \epsilon$. We round the value of $\tilde{\beta}$ to the nearest multiple of $\epsilon$ so that the number of steps for the binary search is bounded by $\log \frac{1}{\epsilon}$. The problem of finding a minimum capacity edge set to decompose a graph of size $n$ into strongly connected components of size at most $\rho n$ is known as $\rho$-separator problem. We use here the algorithm presented in [23] that for a fixed $\epsilon>0$ finds a $\rho$ separator in directed graph $G$ whose value is at most $O\left(\frac{1}{\epsilon^{2}} \cdot \log n \log \log n\right)$ times $\operatorname{Opt}_{(\rho-\epsilon) \text {-separator where }}$

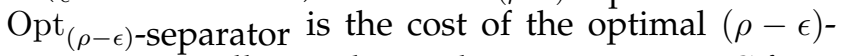
separator. Finally, we derive the cut vertices in $G$ from the cut edges in $G^{\prime}$ to obtain the $\beta^{\prime}$-vertex disruptor.

Lemma 2: Algorithm 3 always terminates with a $\beta^{\prime}$ vertex disruptor.

Proof: We show that whenever $\tilde{\beta} \leq \beta^{\prime}$ then the corresponding $D_{v}$ found in Algorithm 3 is a $\beta^{\prime}$-vertex disruptor in $G$. Consider the edge disruptor $D_{e}^{\prime}$ in $G^{\prime}$ induced by $D_{v}$. We first show the mapping between SCCs in $G_{\left[V \backslash D_{v}\right]}$ and SCCs in $G^{\prime}\left[E^{\prime} \backslash D_{e}^{\prime}\right]$, the graph obtained by removing $D_{e}^{\prime}$ from $G^{\prime}$. Partition the vertex set $V$ of $G$ into: (1) $D_{v}$ : the set of removed nodes (2) $V_{\text {single }}$ : the set of nodes that are not in any cycle i.e. they are SCCs of size one (3) $V_{\text {connected }}$ : union of remaining SCCs that sizes are at least two, say $V_{\text {connected }}=\biguplus_{i=1}^{l} C_{i},\left|C_{i}\right| \geq 2$. Vertices in $V_{\text {connected }}$ belong to at least one cycle in $G$.

We have following corresponding SCCs in $G^{\prime}\left[E^{\prime} \backslash D_{e}^{\prime}\right]$ :

1) $v \in D_{v} \leftrightarrow \operatorname{SCCs}\left\{v^{+}\right\}$and $\left\{v^{-}\right\}$. Since after removing $\left(v^{-} \rightarrow v^{+}\right) v^{+}$does not have incoming edges and $v^{-}$does not have outgoing edges.

2) $v \in V_{\text {single }} \leftrightarrow \mathrm{SCCs}\left\{v^{+}\right\}$and $\left\{v^{-}\right\}$. Since $v$ does not lie on any cycle in $G$. Assume $v^{+}$belong to some SCC of size at least 2 i.e. $v^{+}$lies on some cycle in $G^{\prime}$. Because the only incoming edge to $v^{+}$is from $v^{-}$. It follows that $v^{-}$is preceding $v+$ on that cycle. Let $u^{-}, u^{+}$be the successive vertices of $v^{+}$on that cycle. We have $u$ and $v$ belong to a same SCC in $G$ which yields a contradiction. Similarly, $v^{-}$cannot lie on any cycle in $G^{\prime}$.

3) $\operatorname{SCC} C_{i} \subset V_{\text {connected }} \leftrightarrow \operatorname{SCC} C_{i}^{\prime}=\left\{v^{-}, v^{+} \mid v \in\right.$ $\left.C_{i}\right\}$. This can be shown using a similar argument to that in the case $v \in V_{\text {single }}$.

Since $D_{e}^{\prime}$ is a $\tilde{\beta}$-separator, the sizes of SCCs in $G^{\prime}\left[E^{\prime} \backslash D_{e}^{\prime}\right]$ are at most $\tilde{\beta} 2 n$. It follows that the sizes of SCCs in $G_{\left[V \backslash D_{v}\right]}$ are bounded by $\tilde{\beta} n$. Denote the set of SCCs in $G_{\left[V \backslash D_{v}\right]}$ by $\mathcal{C}$ with the convention that vertices in $D_{v}$ become singleton SCC in $G_{\left[V \backslash D_{v}\right]}$. Thus, we have:

$$
\begin{aligned}
\mathcal{P}\left(G_{\left[V \backslash D_{v}\right]}\right) & =\sum_{C_{i} \in \mathcal{C}}\left(\begin{array}{c}
\left|C_{i}\right| \\
2
\end{array}\right)=\frac{1}{2}\left(\sum_{C_{i} \in \mathcal{C}}\left|C_{i}\right|^{2}-|V|\right) \\
& \left.\leq \frac{1}{2}\left(\sum_{C_{i} \in \mathcal{C}} \tilde{\beta}|V|\right)\left|C_{i}\right|-|V|\right) \\
& =\frac{1}{2}\left(\tilde{\beta}|V|^{2}-|V|\right) \leq \tilde{\beta}\left(\begin{array}{c}
|V| \\
2
\end{array}\right)<\beta^{\prime}\left(\begin{array}{c}
|V| \\
2
\end{array}\right)
\end{aligned}
$$

This guarantees that the binary search always finds a $\beta^{\prime}$-vertex disruptor and completes the proof.

Theorem 5: Algorithm 3 always finds a $\beta^{\prime}$-vertex disruptor whose the size is at most $O(\log n \log \log n)$ times the optimal $\beta$-vertex disruptor for $\beta^{\prime 2}>\beta>0$.

Proof: It follows from the Lemma 2 that Algorithm 3 terminates with a $\beta^{\prime}$-vertex disruptor $D_{v}$. At some step the capacity of $D_{v}$ equals to the capacity of $\tilde{\beta}$ separator $D_{e}^{\prime}$ in $G^{\prime}$ where $\beta$ is at least $\beta^{\prime}-\epsilon$ according to Lemma 2 and the binary search scheme. The cost of the separator is at most $O(\log n \log \log n)$ times the

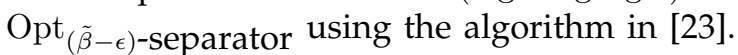

Consider an optimal $\left(\beta^{\prime 2}-9 \epsilon\right)$-vertex disruptor $D_{v}^{\prime}$ of $G$ and its corresponding edge disruptor $D_{e}^{\prime}$ in $G^{\prime}$. Denote the cost of that optimal vertex disruptor by

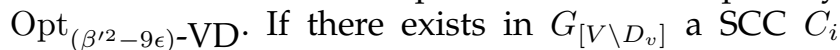
so that $\left|C_{i}\right|>\left(\beta^{\prime}-2 \epsilon\right) n$ then $\mathcal{P}\left(G_{\left[V \backslash D_{v}\right]}\right)>\frac{1}{2}\left(\left(\beta^{\prime}-\right.\right.$ $2 \epsilon) n-2)\left(\left(\beta^{\prime}-2 \epsilon\right) n-1\right)>\left(\beta^{\prime 2}-9 \epsilon\right)\left(\begin{array}{l}n \\ 2\end{array}\right)$ when $n>$ $\frac{20\left(\beta^{\prime}+1\right)}{\epsilon}$. Hence, every SCC in $G_{\left[V \backslash D_{v}^{\prime}\right]}^{\prime}$ have size at most $\left(\beta^{\prime}-2 \epsilon\right)(2 n)$ i.e. $D_{e}^{\prime}$ is an $\left(\beta^{\prime}-2 \epsilon\right)$-separator in $G^{\prime}$. It follows that $\mathrm{Opt}_{\left(\beta^{\prime 2}-9 \epsilon\right)}-\mathrm{VD} \geq \mathrm{Opt}_{\left(\beta^{\prime}-2 \epsilon\right) \text {-separator }}$ in $G^{\prime}$.

Since $\tilde{\beta}-\epsilon \geq \beta^{\prime}-2 \epsilon$, we have $\operatorname{Opt}_{(\tilde{\beta}-\epsilon) \text {-separator }} \leq$ $\mathrm{Opt}_{\left(\beta^{\prime}-2 \epsilon\right) \text {-separator }} \leq \mathrm{Opt}_{\left(\beta^{\prime 2}-9 \epsilon\right)-\mathrm{VD}}$.

The size of the vertex disruptor $\left|D_{v}\right|=\left|D_{e}^{\prime}\right|$ is at most $O(\log n \log \log n)$ times $\mathrm{Opt}_{(\tilde{\beta}-\epsilon) \text {-separator }}$. Thus, the size of found $\beta^{\prime}$-vertex disruptor $D_{v}$ is at most $O(\log n \log \log n)$ times the optimal $\left(\beta^{\prime 2}-9 \epsilon\right)$ vertex disruptor. As we can choose arbitrary small $\epsilon$, setting $\beta=\beta^{\prime 2}-9 \epsilon$ completes the proof.

Time complexity: Finding the separator costs $O\left(n^{9}\right)$ [23]. Hence, the total time complexity is $O\left(\log \frac{1}{\epsilon} n^{9}\right)$. 


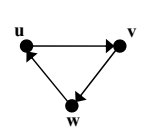

(a)

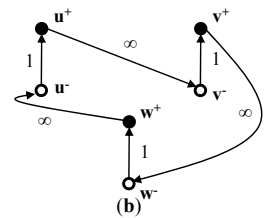

Fig. 4. Conversion from the node version in a directed graph (a) into the edge version in a directed graph (b)

However, in our experiments, the algorithm takes much less than its worst-case running time.

\subsection{Approximating edge disruptor is at least as hard as approximating vertex disruptor}

We show that an approximation algorithm for general directed edge disruptor yields an approximation algorithm for directed vertex disruptor with (almost) the same approximation ratio.

Lemma 3: A $\beta$-edge disruptor set in the directed graph $G^{\prime}$ induces the same cost $\beta$-vertex disruptor set in $G$.

Proof: We use $D_{v}$ and $D_{e}^{\prime}$ for vertex disruptor in $G$ and edge disruptor in $G^{\prime}$.

Given $\mathcal{P}\left(G^{\prime}\left[E^{\prime} \backslash D_{e}^{\prime}\right]\right) \leq \beta\left(\begin{array}{c}2 n \\ 2\end{array}\right)$ we need to prove that: $\mathcal{P}\left(G_{\left[V \backslash D_{v}\right]}\right) \leq \beta\left(\begin{array}{c}n \\ 2\end{array}\right)$ where $n=|V|$.

Assume $G_{\left[V \backslash D_{v}\right]}$ has $l$ SCCs of size at least 2, say $C_{i}, i=1 \ldots l$. The corresponding SCCs in $G^{\prime}\left[E^{\prime} \backslash D_{e}^{\prime}\right]$ will be $C_{i}^{\prime}, i=1 \ldots l$ where $\left|C_{i}^{\prime}\right|=2\left|C_{i}\right|$.

Since $\frac{\left(\begin{array}{c}2 k \\ 2\end{array}\right)}{\left(\begin{array}{c}2 n \\ 2\end{array}\right)}-\frac{\left(\begin{array}{l}k \\ 2\end{array}\right)}{\left(\begin{array}{c}n \\ 2\end{array}\right)}=\frac{k(n-k)}{(n-1) n(2 n-1)} \geq 0$, for all $0 \leq k \leq$ $n$. We have $\frac{\mathcal{P}\left(G_{\left[V \backslash D_{v}\right]}\right)}{\left(\begin{array}{c}n \\ 2\end{array}\right)}=\sum_{i=1}^{l} \frac{\left(\begin{array}{c}\left|C_{i}\right| \\ 2\end{array}\right)}{\left(\begin{array}{c}n \\ 2\end{array}\right)} \leq \sum_{i=1}^{l} \frac{\left(\begin{array}{c}\left|C_{i}^{\prime}\right| \\ 2\end{array}\right)}{\left(\begin{array}{c}2 n \\ 2\end{array}\right)} \leq \beta$

Lemma 4: A $\beta$-vertex disruptor set in $G$ induces the same cost $(\beta+\epsilon)$-edge disruptor set in $G^{\prime}$ for any $\epsilon>0$.

Proof: We use the same notations in the proof of Lemma 3. Given $\mathcal{P}\left(G_{\left[V \backslash D_{v}\right]}\right) \leq \beta\left(\begin{array}{c}n \\ 2\end{array}\right)$ we need to prove that: $\mathcal{P}\left(G^{\prime}\left[E^{\prime} \backslash D_{e}^{\prime}\right]\right) \leq(\beta+\epsilon)\left(\begin{array}{c}2 n \\ 2\end{array}\right)$. We have:

$$
\begin{aligned}
\frac{\mathcal{P}\left(G^{\prime}\left[E^{\prime} \backslash D_{e}^{\prime}\right]\right)}{\left(\begin{array}{c}
2 n \\
2
\end{array}\right)} & =\sum_{i=1}^{l} \frac{\left|C_{i}\right|\left(n-\left|C_{i}\right|\right)}{(n-1) n(2 n-1)}+\frac{\mathcal{P}\left(G_{\left[V \backslash D_{v}\right]}\right)}{\left(\begin{array}{c}
n \\
2
\end{array}\right)} \\
& =\frac{\mathcal{P}\left(G_{\left[V \backslash D_{v}\right]}\right)}{\left(\begin{array}{c}
n \\
2
\end{array}\right)}\left(1-\frac{1}{2 n-1}\right)+\frac{\sum_{i=1}^{l}\left|C_{i}\right|}{n(2 n-1)} \\
& <\beta+\frac{1}{2 n-1}<\beta+\epsilon
\end{aligned}
$$

when $n \geq\left\lfloor\frac{1+\epsilon}{2 \epsilon}\right\rfloor+1$.

Theorem 6: Given a factor $f(n)$ polynomial time approximation algorithm for $\beta$-edge disruptor, there exists a factor $(1+\epsilon) f(n)$ polynomial time approximation algorithm for $\beta$-vertex disruptor where $\epsilon>0$ is an arbitrary small constant.

Proof: Let $G$ be a directed graph with uniform vertex costs in which we wish to find a $\beta$-vertex disruptor. Construct $G^{\prime}$ as described at the beginning of this Section.

Apply the given approximation algorithm to find in $G^{\prime}$ a $\beta$-edge disruptor, denoted by $D_{e}^{\prime}$, with the cost at most $f(n) \cdot \mathrm{Opt}_{\beta-\mathrm{ED}}\left(G^{\prime}\right)$, where $\mathrm{Opt}_{\beta-\mathrm{ED}}\left(G^{\prime}\right)$ is the cost of a minimum $\beta$-edge disruptor in $G^{\prime}$. From
Lemma 3, $D_{e}^{\prime}$ induces in $G$ a $\beta$-vertex disruptor $D_{v}$ of the same cost. We shall prove that

$$
\mathrm{Opt}_{\beta-\mathrm{ED}}\left(G^{\prime}\right) \leq \mathrm{Opt}_{\beta-\mathrm{VD}}(G)+\gamma_{0},
$$

where $\operatorname{Opt}_{\beta-\mathrm{VD}}(G)$ is the cost of a minimum $\beta$-vertex disruptor in $G$ and $\gamma_{0}$ is some positive constant. It follows that the cost of $D_{v}$ will be at most

$$
f(n) \cdot\left(\operatorname{Opt}_{\beta-\mathrm{VD}}(G)+\lambda_{0}\right) \leq(1+\epsilon) f(n) \mathrm{Opt}_{\beta-\mathrm{VD}}(G)
$$

Here, we assume that $\operatorname{Opt}_{\beta-\mathrm{VD}}(G)>\frac{\gamma_{0}}{\epsilon}$ otherwise we can find $\operatorname{Opt}_{\beta-\mathrm{VD}}(G)$ in time $O\left(n^{\frac{\gamma_{0}}{\epsilon}+2}\right)$.

From an optimal $\beta$-vertex disruptor of $G$, construct its corresponding edge disruptor $D_{e}^{*}$ in $G^{\prime}$. If $\mathcal{P}\left(G^{\prime}\left[E \backslash D_{e}^{*}\right] \leq \beta\left(\begin{array}{c}2 n \\ 2\end{array}\right)\right.$ then $\operatorname{Opt}_{\beta-\mathrm{ED}}\left(G^{\prime}\right) \leq$ $\operatorname{Opt}_{\beta-\mathrm{VD}}(G)$ and we yield the proof. Thus, we consider the case $\mathcal{P}\left(G^{\prime}\left[E \backslash D_{e}^{*}\right]>\beta\left(\begin{array}{c}2 n \\ 2\end{array}\right)\right.$.

Among SCCs of $G^{\prime}\left[E \backslash D_{e}^{*}\right]$, there must be a SCC of size at least $\beta 2 n$ or else $G^{\prime}\left[E \backslash D_{e}^{*}\right] \leq \beta^{-1}\left(\begin{array}{c}\beta 2 n \\ 2\end{array}\right) \leq$ $\beta\left(\begin{array}{c}2 n \\ 2\end{array}\right)$ (contradiction). Remove $\gamma_{0}=\left\lceil\frac{1}{\beta}\right\rceil$ vertices from that SCC. The pairwise connectivity in $G^{\prime}\left[E \backslash D_{e}^{*}\right]$ will decrease at least $\left(\beta 2 n-\frac{1}{\beta}\right) \frac{1}{\beta}=2 n-\frac{1}{\beta^{2}} \geq n$ for sufficient large $n$. From Eq. 2 in Lemma 4, the pairwise connectivity after removing vertices will be less than

$$
\left(\beta+\frac{1}{2 n-1}\right)\left(\begin{array}{c}
2 n \\
2
\end{array}\right)-n \leq \beta\left(\begin{array}{c}
2 n \\
2
\end{array}\right) .
$$

Therefore, after removing at most $\gamma_{0}$ vertices from $D_{e}^{*}$, we get a $\beta$-edge disruptor. Hence,

$$
\operatorname{Opt}_{\beta-\mathrm{ED}}\left(G^{\prime}\right) \leq \operatorname{Opt}_{\beta-\mathrm{VD}}(G)+\gamma_{0} \text {. }
$$

\section{EXPERIMENTAL STUDY}

In this section, we discuss a set of experiments designed to gain insight into the performance of the pseudo-approximation in different settings. In the first set of experiments, we compare the performance of the pseudo-approximation algorithm (Algorithm 3) to the performance of an optimal solution obtained by solving an Integer programming formulation of the problem. To this end, we generate two types of networks: random networks, based on Erdos-Rényi model, and power-law networks, based on BarabásiAlbert model. For each type of network, we generate different instances of networks, with a number of nodes ranging from 30 to 100 . The edge densities of the generated networks are around $10 \%$. The experiments were carried out on an an 8-core, $2.2 \mathrm{Ghz}$, machine, and 64 GB memory.

The size of the disruptors found by Algorithm 3 and the optimal disruptors, respectively, are presented in Tables 1 and 2. The results clearly show that the pseudo-approximation algorithm produces nearoptimal solutions in all cases, with an optimal solution in more than $50 \%$ of the cases,shown in boldfaced in the table.

In particular, the results show that our algorithm performs extremely well on power-law networks. It misses the optimal solution in only one case, when the number of vertices is 90 . The results also show 


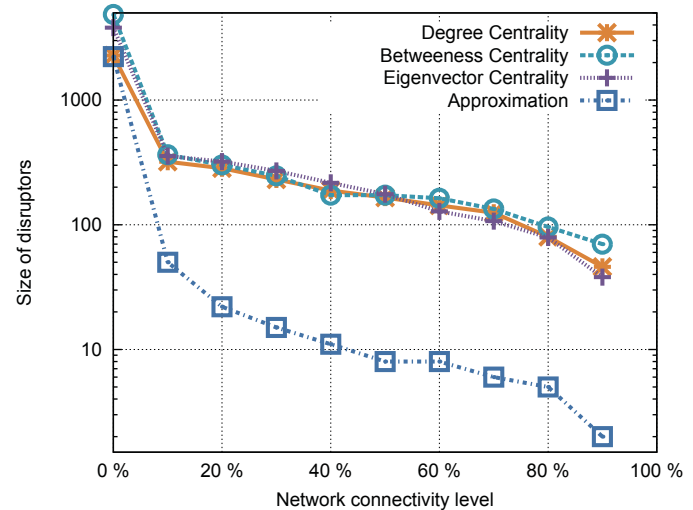

Fig. 5. Disruptors found by different methods in the Western States Power Grid of the United States at different levels of disruption.

that, for a random network and a power-law network of roughly the same size, the size of disruptor in the power-law network is significantly smaller (approximately 50\%) than that in the random network, thereby indicating an extremely high degree of powerlaw network vulnerability to attacks [24]. The running time for solving the Integer programming increases from few minutes to 10 hours for the largest test cases, while in the longest run, the pseudo-approximation algorithm takes only 29 seconds.

TABLE 1

Size of disruptor on Erdos-Rényi networks at $60 \%$ connectivity.

\begin{tabular}{crrrrrrrr}
\hline Vertex & 30 & 40 & 50 & 60 & 70 & 80 & 90 & 100 \\
Edge & 43 & 78 & 122 & 177 & 241 & 316 & 400 & 495 \\
\hline Optimal & 2 & 4 & 7 & 9 & 11 & 12 & 16 & 18 \\
Approx & 3 & 4 & 8 & $\mathbf{9}$ & $\mathbf{1 1}$ & 13 & $\mathbf{1 6}$ & 19 \\
\hline
\end{tabular}

TABLE 2

Size of disruptor on Barabási-Albert networks at $60 \%$ connectivity.

\begin{tabular}{crrrrrrrr}
\hline Vertex & 30 & 40 & 50 & 60 & 70 & 80 & 90 & 100 \\
Edge & 54 & 131 & 189 & 208 & 245 & 262 & 354 & 445 \\
\hline Optimal & 1 & 3 & 5 & 6 & 6 & 5 & 7 & 9 \\
Approx & $\mathbf{1}$ & $\mathbf{3}$ & $\mathbf{5}$ & $\mathbf{6}$ & $\mathbf{6}$ & $\mathbf{5}$ & 10 & $\mathbf{9}$ \\
\hline
\end{tabular}

In the second set of experiments, we study a network of 4941 nodes and 6594 edges representing the topology of the Western States Power Grid of the United States. The network exhibits high clustering with small path lengths, indicating a high vulnerability to attacks [25]. Finding an optimal disruptor for such a large network, using Integer Programming, is clearly intractable. To overcome this shortcoming, an approximation algorithm, which uses a rowgeneration technique to reduce excessive amount of constraints, was developed. Using this algorithm, the experiments were run on a clusters of 20 nodes, where each node is equipped with an 8-core, $2.2 \mathrm{Ghz}$, and 64 GB memory.

In this study, we compare the performance of the node-centrality based schemes to the performance of the proposed 'pseudo-approximation algorithm. Of particular focus is the ability of the scheme to accurately assess the network vulnerability in term of overall network connectivity. The methods used in this comparative study include:

1) Degree Centrality: The algorithm sequentially removes the node with the maximum degree until the pairwise connectivity in the graph becomes less than $\beta\left(\begin{array}{c}n \\ 2\end{array}\right)$.

2) Betweenness Centrality: The algorithm repeatedly removes the node with the maximum betweeness centrality, until the pairwise connectivity in the graph becomes less than $\beta\left(\begin{array}{l}n \\ 2\end{array}\right)$. Recall that the betweeness, $B t(v)$ for node $v$ is expressed as $B t(v)=\sum_{\substack{s \neq v \neq t \in V \\ s \neq t}} \frac{\sigma_{s t}(v)}{\sigma_{s t}}$ where $\sigma_{s t}$ is the number of shortest paths from $s$ to $t$, and $\sigma_{s t}(v)$ is the number of shortest paths from $s$ to $t$ that pass through a node $v$.

3) Eigenvector Centrality: In this algorithm, nodes are removed in descending order of their eigenvector centrality values, using a default damping factor of 0.85 [26].

Figure 5 shows the vulnerability reported by different methods for various levels of disruption. The results show that the network is surprisingly vulnerable to targeted attacks. For example, reducing network connectivity by $40 \%$, only requires the destruction of $0.16 \%$ of the stations. Reducing connectivity in random and power-law networks by the same level, however, requires, on average, the destruction of $13 \%$ and $3 \%$ of the network nodes, respectively. The results also show that the destruction of only $1 \%$ of stations dramatically disrupt network operations and reduces the power grid by $90 \%$.

With regard to assessing overall network vulnerability, the results show that node-centrality based methods perform poorly. None of these methods is able to evaluate correctly the vulnerability of the power grid. Their disruptor sizes are 6 to 20 times larger than the corresponding ones produced by the pseudo-approximation algorithm. This clearly limits their usage in assessing the vulnerability of largescale, critical infrastructure such as the power grid. It is also worth noting that, because of high clustering property, nodes that lie within clusters in the network, often have high betweenness values. Intuitively, it is, therefore, expected the the Betweenness Centrality method would easily identify those nodes as highly risk nodes. Surprisingly, the performance of this method was worse than that of the Degree Centrality method.

\section{Conclusion}

In this paper, a novel framework, based on network pairwise connectivity, to assess network vulnerability is proposed. The framework provides the basis 
to investigate network vulnerability as optimization problem, referred to as the $\beta$-disruptor, whose objective is to determine the the minimum number of network elements - nodes and vertices - that must be removed to reduce the network pairwise connectivity to a specific level. We present hardness results related to this problem, including the NP-Completeness and inapproximability, along with two pseudo-approximation algorithms with provable performance bounds. The accuracy of our framework compared with existing measurements are validated through a series of experiments of both simulated and real networks. The results show that $\beta$-disruptor framework exhibits higher accuracy in assessing network vulnerability than schemes based on node centrality metrics.

\section{REFERENCES}

[1] Tony H. Grubesic, Timothy C. Matisziw, Alan T. Murray, and Diane Snediker. Comparative approaches for assessing network vulnerability. Inter. Regional Sci. Review, 31, 2008.

[2] R. Church, M. Scaparra, and R. Middleton. Identifying critical infrastructure: the median and covering facility interdiction problems. Ann Assoc Am Geogr, 94(3):491-502, 2004.

[3] A. Murray, T. Matisziw, and T. Grubesic. Multimethodological approaches to network vulnerability analysis. Growth Change, 2008.

[4] A. Sen, S. Murthy, and S. Banerjee. Region-based connectivity - a new paradigm for design of fault-tolerant networks. In HPSR, 2009.

[5] S. Neumayer, G. Zussman, R. Cohen, and E. Modiano. Assessing the vulnerability of the fiber infrastructure to disasters. In INFOCOM, 2009.

[6] Charles J. Colbourn. The Combinatorics of Network Reliability. Oxford University Press, Inc., New York, NY, USA, 1987.

[7] Stephen P. Borgatti and Martin G. Everett. A graph-theoretic perspective on centrality. Social Networks, 28(4):466-484, 2006.

[8] D. Goyal and J. Caffery. Partitioning avoidance in mobile ad hoc networks using network survivability concepts. ISCC, page $553,2002$.

[9] M. Hauspie, J. Carle, and D. Simplot. Partition detection in mobile ad hoc networks using multiple disjoint paths set. Workshop of Objects, Models and Multimedia technology, 2003.

[10] M. Jorgic, I. Stojmenovic, M. Hauspie, and D. Simplot-Ryl. Localized algorithms for detection of critical nodes and links for connectivity in ad hoc networks. 3rd IFIP MED-HOC-NET Workshop, 2004.

[11] A. Barabasi, R. Albert, and H. Jeong. Scale-free characteristics of random networks: the topology of the world-wide web. Physica A, 281, 2000.

[12] Y. J. Suh, D. J. Kim, W. S. Lim, and J. Y. Baek. Method for supporting quality of service in heterogeneous networks, 2009.

[13] T. Lehman, J. Sobieski, and B. Jabbari. Dragon: a framework for service provisioning in heterogeneous grid networks. IEEE Communication Magazines, 2006.

[14] V. Mhatre and C. Rosenberg. Homogeneous vs heterogeneous clustered sensor networks: a comparative study. IEEE ICC, 2004.

[15] Fangting Sun and Mark A. Shayman. On pairwise connectivity of wireless multihop networks. International Journal of Security and Networks, 2(1/2):37-49, 2007.

[16] A. Arulselvan, Clayton W. Commander, L. Elefteriadou, and Panos M. Pardalos. Detecting critical nodes in sparse graphs. Computers \& Operations Research, 36(7):2193-2200, 2009.

[17] Stephen P. Borgatti. Identifying sets of key players in a social network. Computational \& Mathematical Organization Theory, 12(1):21-34, 2006.

[18] M. Stoer and F. Wagner. A simple min-cut algorithm. J. of ACM, 44(4):585-591, 1997

[19] D. Wagner and F. Wagner. Between min cut and graph bisection. In MFCS, pages 744-750, London, UK, 1993. SpringerVerlag.
[20] Michael R. Garey and David S. Johnson. Computers and Intractability; A Guide to the Theory of NP-Completeness. W. $\mathrm{H}$. Freeman \& Co., New York, NY, USA, 1990.

[21] I. Dinur and S. Safra. On the hardness of approximating minimum vertex cover. Annals of Mathematics, 162:2005, 2004.

[22] A. Agarwal, M. Charikar, K. Makarychev, and Y. Makarychev. $\mathrm{O}(\log \mathrm{n})$ approximation algorithms for min uncut, $\min 2 \mathrm{cnf}$ deletion, and directed cut problems. In STOC, pages 573-581, New York, NY, USA, 2005. ACM.

[23] G. Even, J. S. Naor, S. Rao, and B. Schieber. Divide-andconquer approximation algorithms via spreading metrics. J. of ACM, 47(4):585-616, 2000.

[24] R. Albert, H. Jeong, and A. Barabasi. Error and attack tolerance of complex networks. Nature, 406(6794):378-382, July 2000.

[25] D. J. Watts and S. H. Strogatz. Collective dynamics of 'smallworld' networks. Nature, 393(6684):440-442, June 1998.

[26] L. Page, S. Brin, R. Motwani, and T. Winograd. The pagerank citation ranking: Bringing order to the web. Technical report, Stanford InfoLab, 1999

Thang $\mathbf{N}$. Dinh received the BA degree in In-

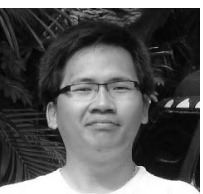
formation Technology from Vietnam National University, Hanoi, Vietnam in 2007 . He is currently a PhD student at the Department of Computer and Information Science and Engineering, University of Florida, under the supervision of Dr. My T. Thai. His research focuses on designing combinatorial optimization methods for dynamic complex networks and mobile ad hoc network including network vulnerability, dynamic community structure, and fast information propagation.

Ying Xuan received the BE degree in computer engineering from the University of Science and Technology of China, Anhui, China, in 2006. He is now a PhD candidate at the Department of Computer and Information Science and Engineering, University of Florida, under the supervision of Dr. My T. Thai. His research topics include applied group testing theory, social networking and network vulnerability.

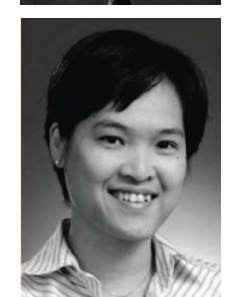

My T. Thai received her PhD degree in computer science from the University of Minnesota, Twin Cities, in 2006. She is an associate professor in the Department of Computer and Information Sciences and Engineering at the University of Florida. Her current research interests include algorithms and optimization on network science and engineering She also serves as an associate editor for the Journal of Combinatorial Optimization (JOCO) and Optimization Letters and a conference chair of $\mathrm{CO}$ COON 2010 and several workshops in an area of network science. She is a recipient of DoD Young Investigator Awards and NSF CAREER awards. She is a member of the IEEE.

Panos M. Pardalos is a Distinguished Profes-

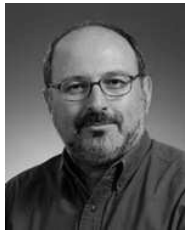
sor of Industrial and Systems Engineering at the University of Florida. He is the director of the Center for Applied Optimization. Dr. Pardalos obtained a $\mathrm{PhD}$ degree from the University of Minnesota in Computer and Information Sciences. Dr. Pardalos is the editor-in-chief of the "Journal of Global Optimization," and of the journals "Optimization Letters," "Computational Management Science," and "Energy Systems."

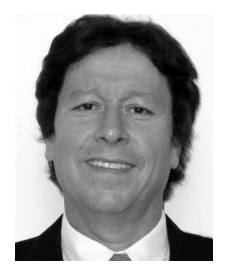

Taieb Znati Taieb Znati received the MS degree in computer science from Purdue University in 1984 , and the $\mathrm{PhD}$ degree in computer science from Michigan State University in 1988. He is a professor in the Department of Computer Science, with a joint appointment in Telecommunications in the Department of Information Science, University of Pittsburgh. He currently serves as the director of the Computer and Network Systems (CNS) Division at the National Science Foundation (NSF). From 2000 to 2004, he served as a senior program director for networking research at NSF. He also served as the committee chairperson of the Information Technology Research (ITR) Program and an NSF-wide research initiative in information technology. His current research interests are on network science and engineering, with the focus on the design of scalable, robust, and reliable network architectures and protocols for wired and wireless communication networks. He is a recipient of several research grants from government agencies and from industry. $\mathrm{He}$ is frequently invited to present keynotes in networking and distributed conferences both in the United States and abroad. He is a member of the IEEE. 